\title{
Effect of Predictive Nursing Intervention on Prevention of Nocturnal Hypoglycemia in Patient with Liver Cirrhosis
}

\author{
Jieyun Luo, Guiyan Wen* \\ Email address: \\ Ljy156527@qq.com (Jieyun Luo),wengy78@126.com (Guiyan Wen) \\ ${ }^{*}$ Corresponding author
}

Gastroenterology Department, The First Affiliated Hospital of Jinan University, Guangzhou, China

\section{To cite this article:}

Jieyun Luo, Guiyan Wen. Effect of Predictive Nursing Intervention on Prevention of Nocturnal Hypoglycemia in Patient with Liver Cirrhosis. American Journal of Nursing Science. Vol. 10, No. 2, 2021, pp. 138-141. doi: 10.11648/j.ajns.20211002.15

Received: March 9, 2021; Accepted: March 19, 2021; Published: March 30, 2021

\begin{abstract}
Background: liver cirrhosis is often accompanied by disease in nocturnal hypoglycemia as the capacity of the liver to preserve carbohydrates is impaired. nocturnal hypoglycemia not only is a poor factor, but also is an indicator of malnutrition assessment in patient with liver cirrhosis. Objective: To evaluate effect of predictive nursing intervention on prevention of nocturnal hypoglycemia in patient with Liver cirrhosis. Methods: From April 2018 to December 2020, we collect valid data from 80 patients. the participants were divided into control group and intervention group: control group received common nursing intervention. intervention group participants received predictive nursing intervention. As for collecting, basic information of participant was collected by participants' medical records and doctor's diagnosis. During study period, we collected frequency of hypoglycemia and liver function data before and after nursing intervention. Rsult: In patient characteristics research, the factors such as gender, age, Child-Pugh assessment and course of the disease were no significant difference between intervention group and control group. There had 49 females $26(65.0 \%)$ in intervention group and $23(57.5 \%)$ in control group] and 31 males 14 $(35.0 \%)$ in intervention group and $17(42.5 \%)$ in control group]. Additionally, intervention group participants had higher blood glucose index compare with control group participants as the results were significant difference between the two groups. In the research of liver function index, predictive nursing intervention improve the liver function index, even its effect was better than common nursing intervention. Conclusion: the effect of improving hypoglycemia in patient with Liver cirrhosis was better among intervention group compare to control group.
\end{abstract}

Keywords: Hypoglycemia, Liver Cirrhosis, Nursing

\section{Introduction}

The liver cirrhosis is basic on the end stage of various liver cirrhosis diseases, it is usually characterized by chronic, progressive and diffuse liver injury [1]. According to Schuppan's report, global incidence of liver cirrhosis was approximately $1 \%$ to $2 \%$ [2]. In addition, liver cirrhosis is often accompanied by disease in nocturnal hypoglycemia as the capacity of the liver to preserve carbohydrates is impaired $[3,4]$. nocturnal hypoglycemia not only is a poor factor, but also is an indicator of malnutrition assessment in patient with liver cirrhosis $[5,6]$. Therefore, exploring the method of improving nocturnal hypoglycemia in patient with chronic liver will be beneficial for treatment effect of liver cirrhosis, reducing mortality, and improving quality of life.

Base on different researches, the outcome of improved hypoglycemia in patients with cirrhosis is different. These studies attempt to use different nursing interventions to improve the hypoglycemia of patients with cirrhosis [7-9]. Of those, nursing intervention has been shown to be important, because nursing intervention is a key factor of treatment outcome changing. Some researches show that nursing intervention has achieved good results in liver cirrhosis patient groups. For instance, Nayan's report indicates comprehensive nursing intervention can improve the problem of hypoglycemia [10]. Therefore, the effect of different nursing intervention on patient with Liver cirrhosis is worth study. The aim of this study was to assess effect of predictive nursing intervention on prevention of nocturnal hypoglycemia in patient with Liver cirrhosis. 


\section{Methods}

\subsection{Participants Enrollment and Survey Methods}

102 patients who were diagnosed as liver cirrhosis were invited to join our study, but 22 patients lacked valid data, so the researchers only had valid data from 80 patients. All participants received treatment from April 2018 to December 2020, and All of them hospitalized in the First Affiliated Hospital of Jinan University. According to receiving nursing intervention, the participants were divided into control group and intervention group: control group received common nursing intervention, intervention group participants received predictive nursing intervention. As for collecting, basic information of participant was collected by participants' medical records and doctor's diagnosis. On the other hand, our researchers collected frequency of hypoglycemia and liver function data before and after nursing intervention.

As for nursing intervention, we provided predictive nursing intervention to intervention group participants. Details of the predictive nursing intervention include the following: (1) Nurses are concerned about the risk of hypoglycemia by training; (2) We provide sugar or dextrose solution to participants during periods when food and water are forbidden; (3) We regularly monitored the participants' glycemic index, and we regularly observe the participants' reactions.

Participants' inclusion criteria include: (1) patients were diagnosed as liver cirrhosis; (2) patients need to be hospitalized; (3) patients volunteered to participate in this study and they agreed to sign the informed consent. Participants' withdraw criteria contrast: (1) patient had other complications; (2) mental state of the patient is poor; (3) patients changed hospitals during the study period.

\subsection{Statistical Analysis}

We use SPSS 22.0 to analyze the data, that we analyze correlation among chi-square test and collection result. Besides, statistical description includes mean and standard deviation.

\section{Result}

Table 1 blew shows that 80 patients who were diagnosed as liver cirrhosis for 32 months were included in this study: The factors such as gender, age, Child-Pugh assessment and course of the disease were no significant difference between intervention group and control group. There had 49 females 26 $(65.0 \%)$ in intervention group and $23(57.5 \%)$ in control group] and 31 males $14(35.0 \%)$ in intervention group and 17 (42.5\%) in control group]. Their age was present in $36.65 \pm 14.23$ and $39.77 \pm 12.87$ years of intervention group, control group. Child-Pugh of two groups were B level, their assessment were $7.55 \pm 3.90$ and $7.94 \pm 4.16$. Most patients with liver cirrhosis had treatment records for half years, their courses of the disease were $5.75 \pm 4.01$ and $5.91 \pm 4.95$ months in intervention group participants, control group participants.

Table 1. Characteristics of participants.

\begin{tabular}{lllll}
\hline & Gender (Female) & Age (year) & Child-Pugh Assessment (Mean \pm SD) & Course of the disease (month) \\
\hline Intervention group $(\mathrm{n}=40)$ & $26(65.0 \%)$ & $36.65 \pm 14.23$ & $7.55 \pm 3.90$ & $5.75 \pm 4.01$ \\
Control group $(\mathrm{n}=40)$ & $23(57.5 \%)$ & $39.77 \pm 12.87$ & $7.94 \pm 4.16$ & $5.91 \pm 4.95$ \\
P value & $>0.05$ & $>0.05$ & $>0.05$ & $>0.05$ \\
\hline
\end{tabular}

Table 2. Blood glucose index and hypoglycemia research.

\begin{tabular}{|c|c|c|c|c|}
\hline & $\begin{array}{l}\text { Average blood glucose } \\
\text { before night (mmol/L) }\end{array}$ & $\begin{array}{l}\text { Average blood glucose } \\
\text { during night }(\mathrm{mmol} / \mathrm{L})\end{array}$ & $\begin{array}{l}\text { Cases of hypoglycemia } \\
\text { during night (case) }\end{array}$ & $\begin{array}{l}\text { Hypoglycemia Rate } \\
\text { during night }(\%)\end{array}$ \\
\hline Intervention group $(n=40)$ & $2.97 \pm 1.03$ & $4.03 \pm 1.25$ & 18 & $45.0 \%$ \\
\hline Control group $(n=40)$ & $3.04 \pm 0.98$ & $3.25 \pm 1.14$ & 27 & $67.5 \%$ \\
\hline $\mathrm{t}$ & 0.34 & 7.49 & - & - \\
\hline$P$ value & $>0.05$ & $<0.05$ & - & - \\
\hline
\end{tabular}

Table 3. Liver function index.

\begin{tabular}{lccc}
\hline & ALT (U/L) & TBIL (U/L) & ALB (g/L) \\
\hline Intervention group $(\mathrm{n}=40)$ & & & \\
BI & $172.64 \pm 32.48$ & $74.84 \pm 3.58$ & $27.11 \pm 5.06$ \\
AI & $44.31 \pm 14.23^{\text {ab }}$ & $16.48 \pm 3.18^{\text {ab }}$ & $35.47 \pm 3.78^{\text {ab }}$ \\
Control group ( $=40)$ & & & $0.88 \pm 0.45$ \\
BI & $171.81 \pm 32.18$ & $74.62 \pm 3.72$ & $25.35 \pm 5.15$ \\
AI & $58.68 \pm 16.46^{\mathrm{a}}$ & $20.72 \pm 3.35^{\mathrm{a}}$ & $31.46 \pm 3.14^{\mathrm{a}}$ \\
\hline
\end{tabular}

Before Intervention $=\mathrm{BI}$

After Intervention $=\mathrm{AI}$

Alanine aminotransferase $=$ ALT

total bilirubin $=$ TBIL

albumin $=$ ALB

albumin/globulin $=\mathrm{A} / \mathrm{G}$

${ }^{a}=$ Compared with the same group after intervention $\mathrm{p}<0.05$

$\mathrm{b}=$ Compared with the control group after intervention $\mathrm{p}<0.05$ 
Independent $\mathrm{T}$ test was used to study the comparison of blood glucose before night and blood glucose during night and the results were as follows: intervention group participants had higher blood glucose index compare with control group participants as the results were significant difference between the two groups (Table 2). Besides, participants' blood glucose indexes were similar before night as there was no significant difference between their result. In hypoglycemia rate, hypoglycemia rate was lower among intervention group compare to control group ( $45.0 \%$ vs $67.5 \%$ ).

There was effect of predictive nursing intervention on liver function index, such as alanine aminotransferase (ALT), total bilirubin (TBIL), albumin (ALB) and albumin/globulin (A/G) (Table 3). In control group, common nursing intervention improve liver function index of participants that include ALT, TBIL, ALB and $\mathrm{A} / \mathrm{G}$. In addition, predictive nursing intervention also improve the liver function index, even its effect was better than common nursing intervention.

\section{Discussion}

The primary aim of the present study was to analyze effect of predictive nursing intervention on prevention of nocturnal hypoglycemia in patient with liver cirrhosis by comparison of predictive nursing intervention and common nursing intervention. In current study, Hagel report that hypoglycemia was the most common type of glucose disturbance and significantly associated with increased mortality and decreased survival in patients with acutely decompensated liver cirrhosis [11]. Of those, almost $30 \%$ of population had an acute glucose disturbance in patient with liver cirrhosis, so improving risk of hypoglycemia is a significative measure for treatment of liver cirrhosis [12-14]. As for Hagel's research, he focused on abnormal glucose tolerance as a predictor of 30-day mortality in patients with decompensated liver cirrhosis [15]. But none of these studies evaluated the effect of nursing intervention on hypoglycemia. Therefore, we explored the effect of nursing intervention on hypoglycemia by this study.

This study shows that predictive nursing intervention can improve nocturnal hypoglycemia in patient with Liver cirrhosis. The participants who received predictive nursing intervention not only had better blood glucose index but also had less cases of hypoglycemia in study. Although some of the glycemic indexes remain at levels that are at risk for hypoglycemia. Besides, predictive nursing intervention had better effect compare with common nursing intervention as liver function index was significant difference between intervention group and control group. Of those, common nursing intervention can improve hypoglycemia in patient with Liver cirrhosis, but predictive nursing intervention had better effect compare with common nursing intervention.

\section{Conclusion}

Overall, the effect of improving hypoglycemia in patient with Liver cirrhosis was better among intervention group compare to control group. Intervention group had better blood glucose index that was significantly higher than control group. Intervention group also improve liver function, that its effect was better than common nursing intervention.

\section{References}

[1] Yao XX, Jiang SL, Yao DM: Current research of hepatic cirrhosis in China. World journal of gastroenterology. 2015; 11 (5): 617-622.

[2] Schuppan D, Afdhal NH: Liver cirrhosis. Lancet (London, England). 2008, 371 (9615): 838-851.

[3] Montano-Loza AJ. Muscle wasting: a nutritional criterion to prioritize patients for liver transplantation. Curr Opin Clin Nutr Metab Care. 2016; 17: 219-225.

[4] Kalafateli M, Konstantakis C, Thomopoulos K, Triantos C. Impact of muscle wasting on survival in patients with liver cirrhosis. World J Gastroenterol. 2015; 21: 7357-7361.

[5] Garcia-Compean D, Jaquez-Quintana JO, Gonzalez-Gonzalez JA, Maldonado-Garza H. Liver cirrhosis and diabetes: risk factors, pathophysiology, clinical implications and management. World J Gastroenterol. 2019; 15 (3): 280-288.

[6] Nishida T, Tsuji S, Tsujii M, Arimitsu S, Haruna Y, Imano E, et al. Oral glucose tolerance test predicts prognosis of patients with liver cirrhosis. Am J Gastroenterol. 2016; 101 (1): 70-75.

[7] Younossi ZM, Koenig AB, Abdelatif D, Fazel Y, Henry L, Wymer M: Global Epidemiology of Nonalcoholic Fatty Liver Disease-Meta-Analytic Assessment of Prevalence, Incidence, and Outcomes. Hepatology. 2016; 64 (1): 73-84.

[8] Zelniker TA, Wiviott SD, Raz I, Im K, Goodrich EL, Bonaca MP, Mosenzon O, Kato ET, Cahn A, Furtado RHM et al: SGLT2 inhibitors for primary and secondary prevention of cardiovascular and renal outcomes in type 2 diabetes: a systematic review and meta-analysis of cardiovascular outcome trials. Lancet. 2019; 393 (10166): 31-39.

[9] Olson JC, Wendon JA, Kramer DJ, Arroyo V, Jalan R, Garcia-Tsao G, et al. Intensive care of the patient with cirrhosis. Hepatology. 2016; 54 (5): 1864-1872.

[10] Na Y. Study on the effect of predictive nursing on the incidence of acute hypoglycemia in patients with cirrhosis complicated with upper gastrointestinal bleeding. China Modern Drug Application. 2018; 12 (01): 168-169.

[11] Malmberg K, Ryden L, Wedel H, Birkeland K, Bootsma A, Dickstein K, et al. Intense metabolic control by means of insulin in patients with diabetes mellitus and acute myocardial infarction (DIGAMI 2): effects on mortality and morbidity. Eur Heart J. 2015; 26 (7): 650-661.

[12] Chen H, Liu L, Qi X, He C, Wu F, Fan D, Han G: Efficacy and safety of anticoagulation in more advanced portal vein thrombosis in patients with liver cirrhosis. European Journal of Gastroenterology \& Hepatology. 2016; 28 (1): 82-89.

[13] Huang YW, Wang TC, Lin SC, Chang HY, Chen DS, Hu JT, et al. Increased risk of cirrhosis and its decompensation in chronic hepatitis B patients with newly diagnosed diabetes: a nationwide cohort study. Clin Infect Dis. 2019; 57 (12): 1695-702. 
[14] Patel A, Mac Mahon S, Chalmers J, Neal B, Billot L, Woodward $\mathrm{M}$, et al. Intensive blood glucose control and vascular outcomes in patients with type 2 diabetes. $\mathrm{N}$ Engl $\mathbf{J}$ Med. 2018; 358 (24): 2560-2572.
[15] Hagel S, Bruns T, Herrmann A, Stallmach A, Schmidt C. Abnormal glucose tolerance: a predictor of 30-day mortality in patients with decompensated liver cirrhosis. Z Gastroenterol. 2017; 49 (3): 331-334. 